\title{
A Case of Achieving Partial Remission with the Combination of Sorafenib and Nivolumab in a Patient with Hepatocellular Carcinoma Showing Dis- ease Progression after Nivolumab Therapy
}

\author{
Sang Youn Hwang', Seon-Mi Lee', Jung Woo Im¹, Ki Jeong Jeon', Cheol-Won Choi ${ }^{2}$, Kyung-Su Kim², Wan Jeon² \\ Departments of ${ }^{1}$ Internal Medicine and ${ }^{2}$ Radiation Oncology, Dongnam Institute of Radiological \& Medical Sciences, Busan, Korea
}

Received Feb. 7, 2019

Revised Feb. 22, 2019

Accepted Feb. 25, 2019
Sorafenib is a well-known approved systemic therapeutic agent used in patients with advanced hepatocellular carcinoma (HCC). Regorafenib and nivolumab are approved as second-line therapeutic drugs in patients showing disease progression after sorafenib therapy. However, there is no established third- or fourth-line therapy in patients with progression after regorafenib or nivolumab treatment. Recently, the combination of tyrosine kinase inhibitors (TKIs) and immune checkpoint inhibitors (ICPIs) has been attempted as a firstline treatment strategy in advanced HCC patients based on the hypothesis that combination therapy may overcome resistance in ICPI monotherapy. On the basis of this suggestion, we herein describe the case of an $\mathrm{HCC}$ patient demonstrating macrovascular invasion, whereby partial remission was achieved via the combination of sorafenib and nivolumab following disease progression after nivolumab therapy. Further studies on the combination of TKIs and ICPIs are necessary to determine ways to manage HCC patients showing disease progression after ICPI therapy. (J Liver Cancer 2019;19:74-78)

Keywords: Hepatocellular carcinoma; Sorafenib; Nivolumab

\section{INTRODUCTION}

Advanced hepatocellular carcinoma (HCC) patients have poor prognosis and, especially, those with portal vein invasion have a survival time of 4 to 8 months. ${ }^{1,2}$ Sorafenib is the most well-known first-line therapeutic agent against advanced HCC, along with tyrosine kinase inhibitors (TKIs) such as regorafenib, cabozantinib, and ramucirumab and immune checkpoint inhibitors (ICPIs) such as anti-programmed cell

\section{Corresponding author : Sang Youn Hwang}

Department of Internal Medicine, Dongnam Institute of Radiological \& Medical Sciences, 40 Jwadong-gil, Jangan-eup, Gijang-gun, Busan 46033, Korea

Tel. +82-51-720-5114, Fax. +82-51-720-5992

E-mail; mongmani@hanmail.net

https://orcid.org/0000-0003-2750-9379 death (PD)-1 or ligand (PDL)-1 inhibitor. Nivolumab is applied as a second-line therapeutic drug in patients showing disease progression after sorafenib treatment. ${ }^{3,4}$ However, there is no proven and effective third- or fourth-line treatment method in patients showing disease progression after regorafenib or nivolumab administration. Recently, it has been reported that the combination of TKIs and ICPIs may overcome resistance in ICPI monotherapy based on the hypothesis that TKIs may have immune-modulating effects such as promoting intra tumoral $\mathrm{T}$ cell infiltration or enhancing tumor antigenicity. ${ }^{5}$ According to these suggestions, we report a case whereby partial remission was achieved via the combination of sorafenib and nivolumab in an HCC patient showing disease progression after nivolumab therapy. 


\section{CASE REPORT}

A 63-year-old male patient was transferred to our hospital for further evaluation of multiple hepatic masses (three lesions, maximal diameter: $6.5 \mathrm{~cm}$ ) that were found in computed tomography (CT) scans at a local clinic. The initial laboratory test showed a hemoglobin count of $12.0 \mathrm{~g} / \mathrm{dL}$, a platelet of count $241 \times 10^{6} / \mathrm{L}$, and a white blood cell count of $6.6 \times 10^{9} / \mathrm{L}$. The liver function test results were within normal limits. The level of alpha-fetoprotein (AFP) was $2.5 \mathrm{ng} / \mathrm{mL}$ and that of protein induced by vitamin $\mathrm{K}$ absence (PIVKA) was $221 \mathrm{mAU} / \mathrm{mL}$. The patient was diagnosed with HCC, Barcelona liver cancer clinic stage B. Transarterial chemoembolization (TACE) was performed three times during of 4 months and we continuously evaluated tumor response using the modified response evaluation criteria in solid tumors. Multiple new recurrences were observed continuously, and we identified this situation as TACE refractoriness and administered $800 \mathrm{mg}$ of sorafenib per day for 3 months. However, the number of intrahepatic tumors increased and new onset portal vein tumor thrombosis (PVTT) was observed in follow-up CT scans. We then performed radiation therapy for PVTT (40 gray/16 fractions) and administered regorafenib at $160 \mathrm{mg} /$ day every 3 weeks for 3 months, but intrahepatic tumors continued to increase in follow-up CT scans. We then administered nivolumab at $3 \mathrm{mg} / \mathrm{kg}$ every 2 weeks for 2 months (1st-4th cycles) and as a result, the development of intrahepatic tumors was decreased. New onset inferior vena cava tumor thrombosis (IVCTT) was observed in follow-up CT scans (Fig. 1). We concluded that this was a result of an unconfirmed immune progressive disease because the levels of AFP (59.7 ng/mL to $47.4 \mathrm{ng} / \mathrm{mL}$ ) and PIVKA $(73,894 \mathrm{mAU} / \mathrm{mL}$ to $43,475 \mathrm{mAU} / \mathrm{mL})$ were decreased. We then performed radiation therapy for IVCTT (20 gray/10 fractions) (Fig. 2) and we applied nivolumab for two additional months (5th-8th cycles). The follow-up CT scans showed that intrahepatic tumors were enhanced and IVCTT progressed. The levels of both AFP (47.4 ng/mL to $374.4 \mathrm{ng} / \mathrm{mL})$ and PIVKA (43,475 mAU/mL to $70,997 \mathrm{mAU} / \mathrm{mL})$ were re-increased (Fig. 3). We identified this situation as true progressive disease, and we applied combination therapy of nivolumab (9th-11th cycles) and sorafenib (400 mg per day) for 2 months. In the follow-up CT scans, intrahepatic tumors and IVCTT were markedly attenuated (maximal change $10 \mathrm{~cm}$ to $6 \mathrm{~cm})$ and the levels of AFP $(374.4 \mathrm{ng} / \mathrm{mL}$ to $229.9 \mathrm{ng} / \mathrm{mL})$ and PIVKA $(70,997 \mathrm{mAU} / \mathrm{mL}$ to $14,742 \mathrm{mAU} / \mathrm{mL})$ were decreased (Fig. 4). The patient received combination treatment continuously and was followed up carefully (The entire clinical course is described in Fig. 5 based on tumor markers and received treatments).

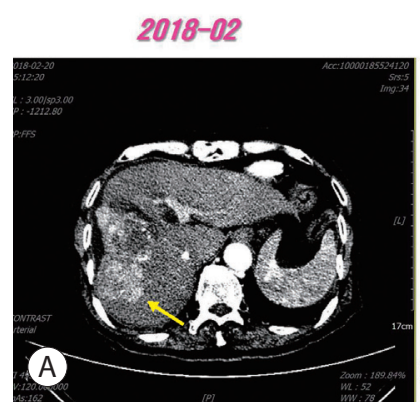

2018-02

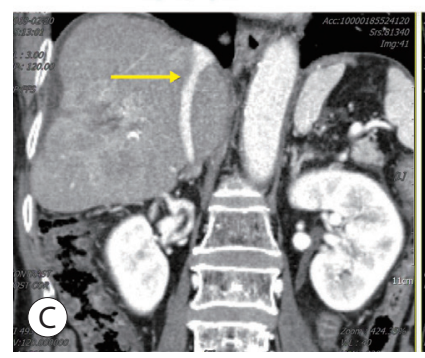

2018-05

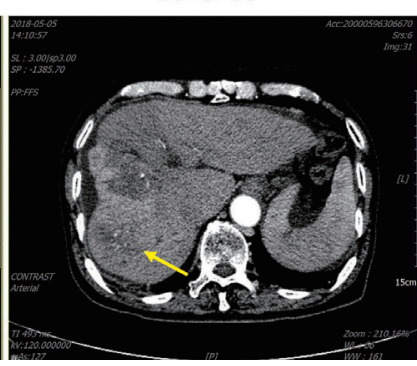

2018-05

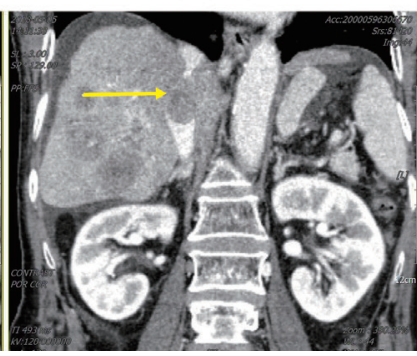

2018-02

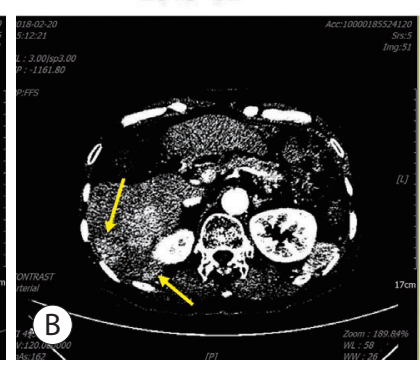

Figure 1. (A, B) Arterial enhancement of intrahepatic tumors was decreased compared to those observed in previous computed tomography scan (arrows). (C) New onset inferior vena cava tumor thrombosis was also observed (arrows). 

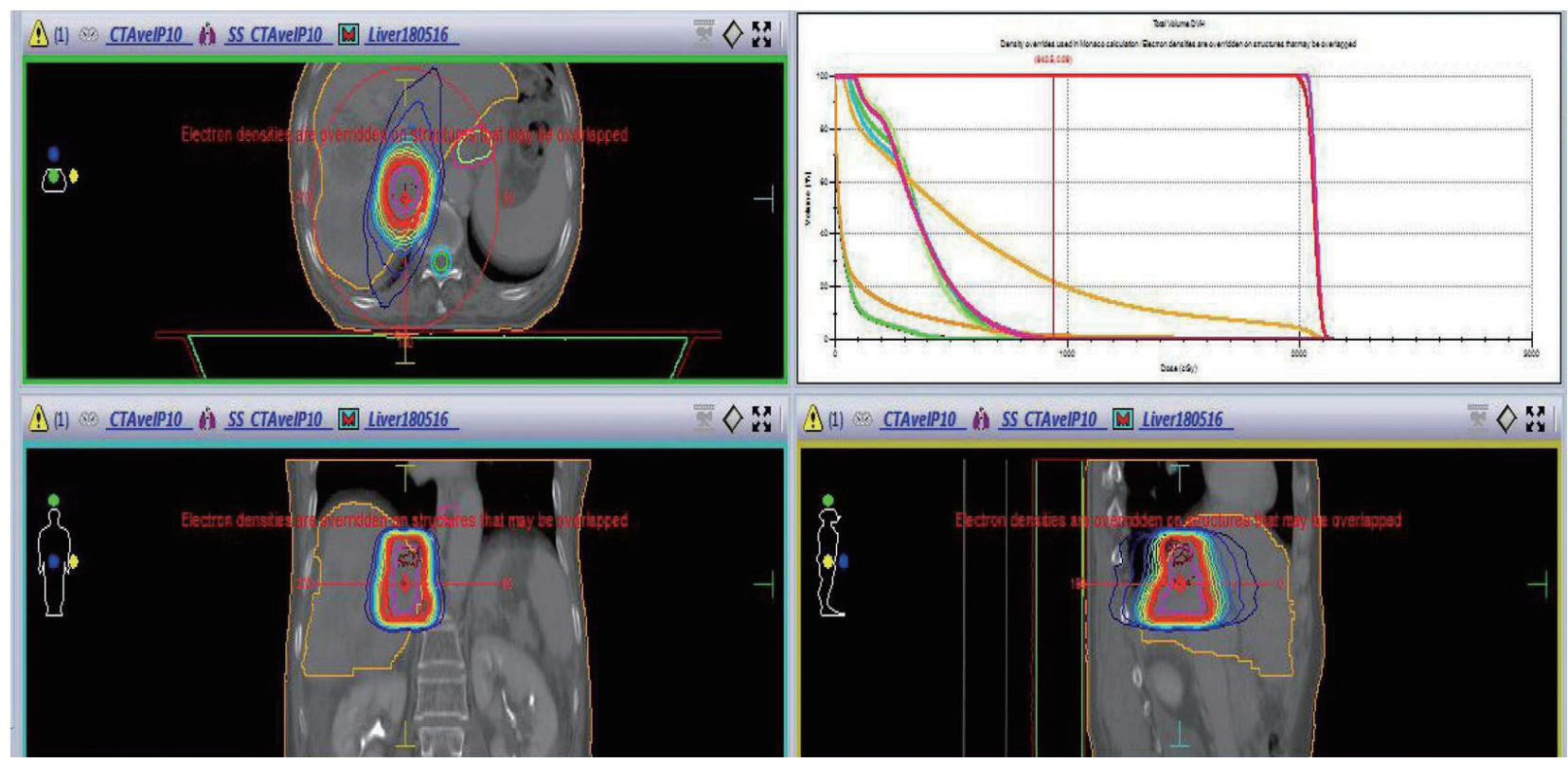

(1) CTAvelP10 it SS CTAvelP10 $\underline{\text { Liver180516 }}$

$\bar{\nabla} \Delta \boldsymbol{M}$

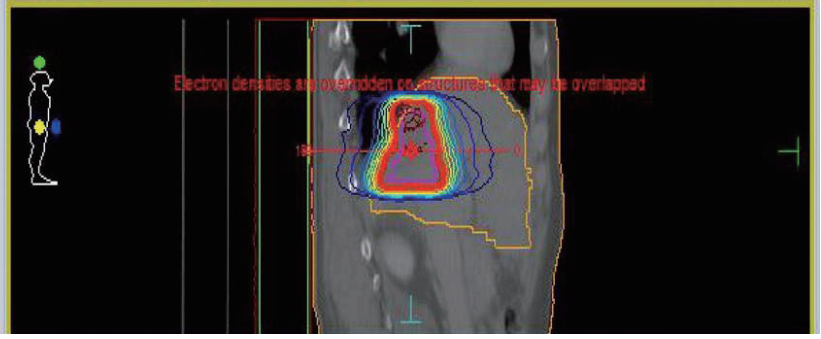

Figure 2. Simulation and planning image of radiation therapy for inferior vena cava tumor thrombosis.

2018-05

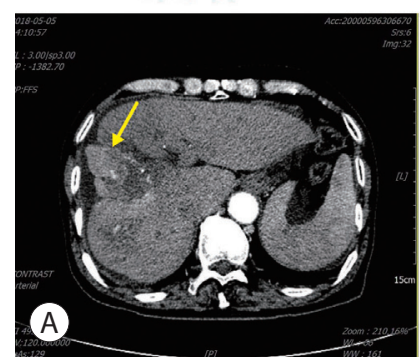

2018-05

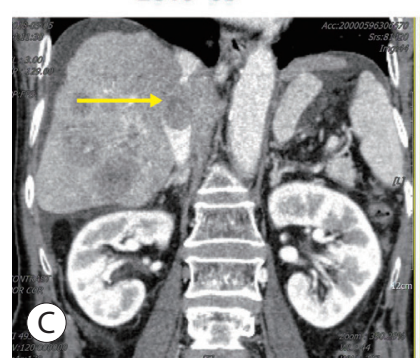

2018-08

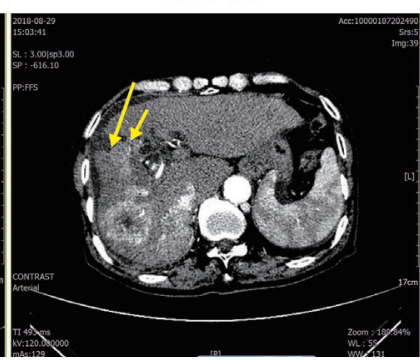

2018-08

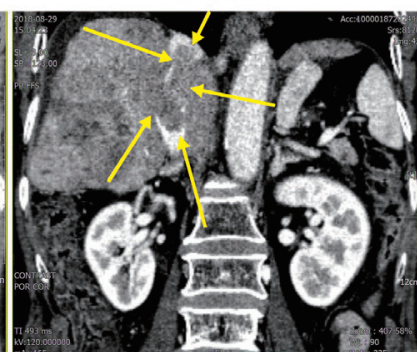

2018-05

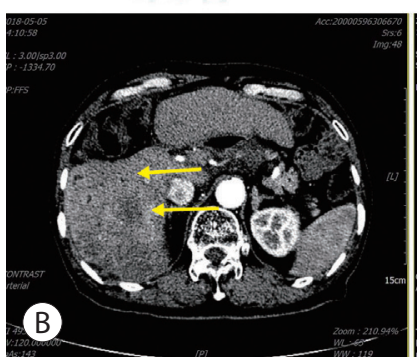

2018-08

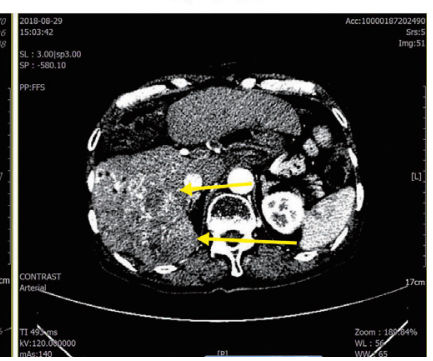

Figure 3. (A, B) Arterial enhancement of intrahepatic tumors was reincreased compared to those observed in previous computed tomography scan (arrows). (C) Progression of inferior vena cava tumor thrombosis was also observed (arrows).

\section{DISCUSSION}

Sorafenib is the most well-known first-line systemic therapeutic against for advanced HCC patients and showed an improved median survival (MS) of 6.5-10.7 months compared to that of the placebo (4.2-6.5 months) ${ }^{1,2}$ Recently, lenvatinib has been approved as a first-line therapeutic

drug and showed an equipotent MS of 13.6 months compared to that of sorafenib (12.3 months). ${ }^{6}$ Regorafenib was approved as a second-line therapeutic agent for patients showing disease progression after sorafenib therapy and showed an improved MS of 10.6 months compared to that of the placebo (7.8 months). ${ }^{3}$ Although CheckMate-040 was a phase II trial, nivolumab was approved as a second-line therapeutic agent 

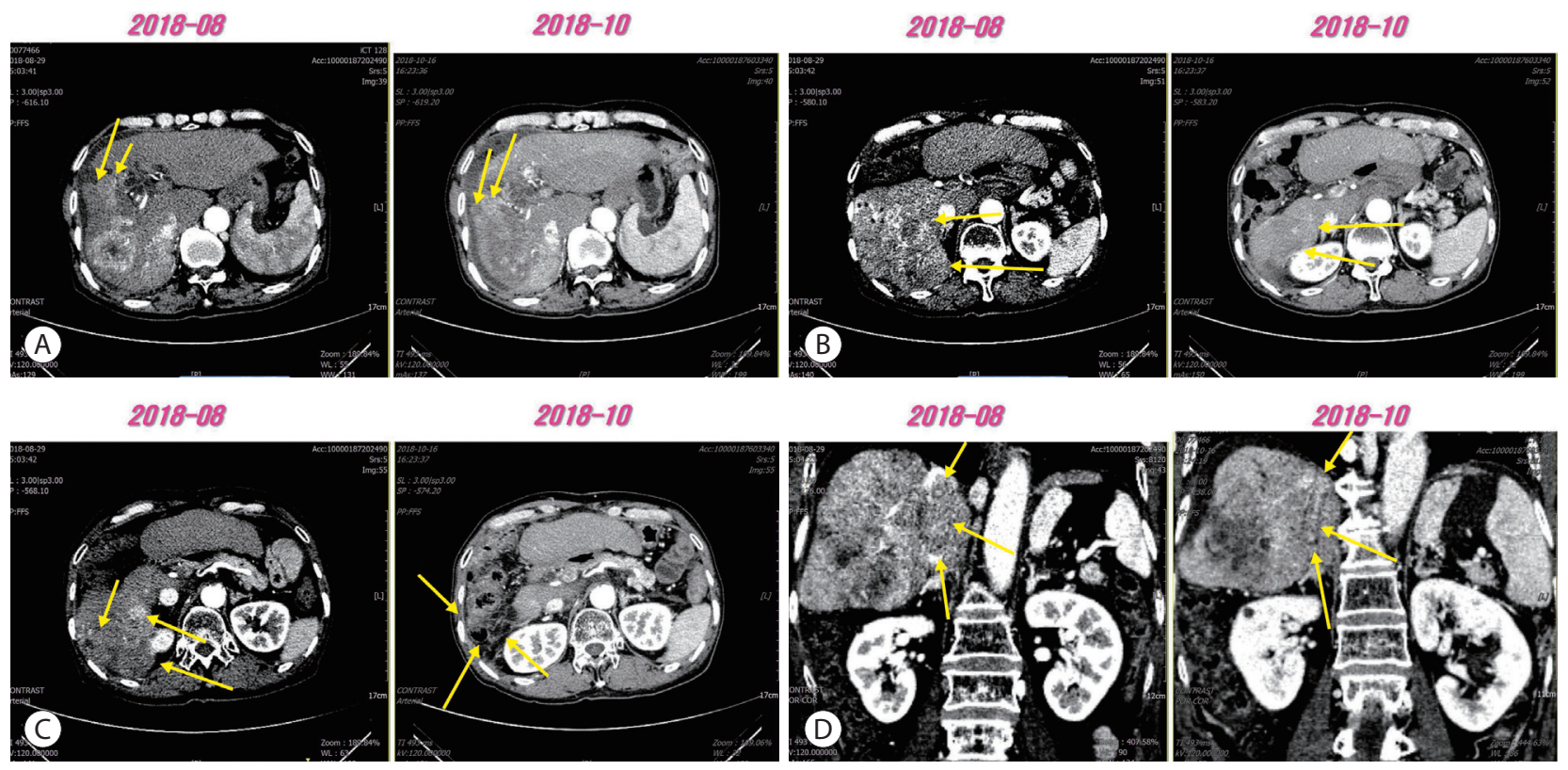

Figure 4. (A-C) Marked shrinkage of intrahepatic tumors was observed compared to those observed in previous computed tomography scan (arrows). (D) Shrinkage of inferior vena cava tumor thrombosis was also observed (arrows).

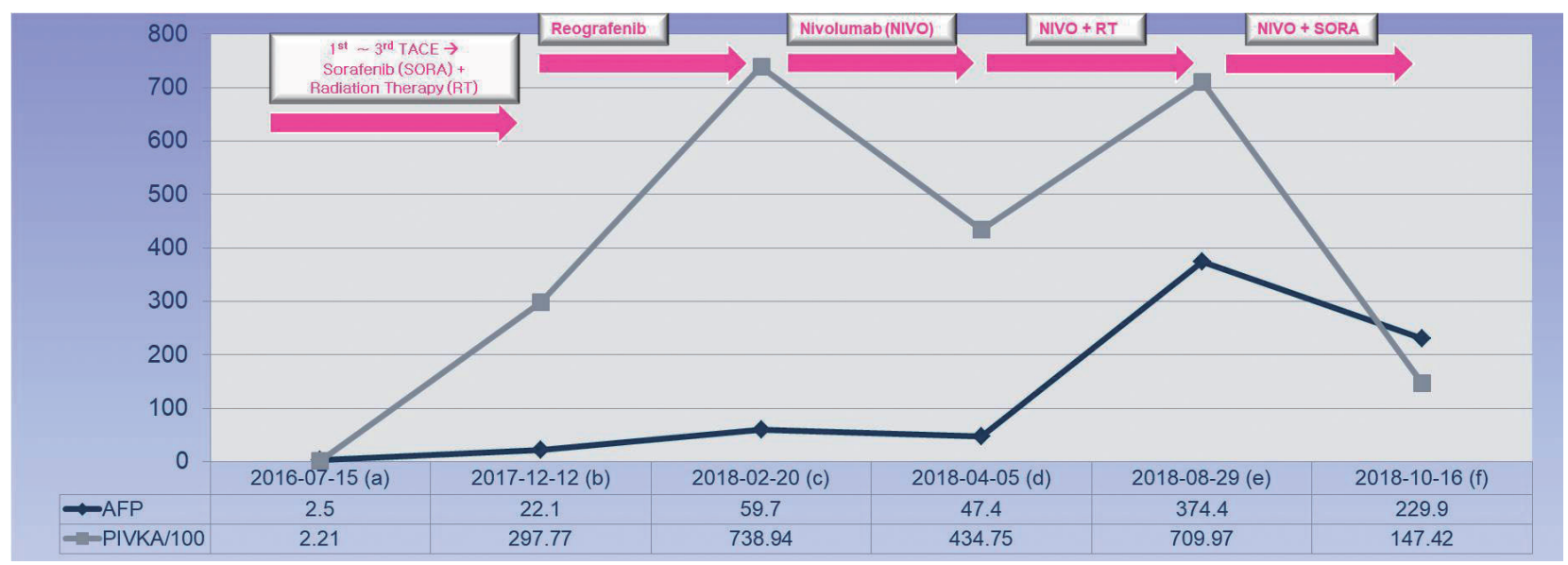

Figure 5. Entire clinical course described by tumor markers and received treatments. TACE, transarterial chemoembolization; AFP, alphafetoprotein; PIVKA, protein induced by vitamin K absence.

for patients showing disease progression after sorafenib therapy because of its noticeable efficacy (MS of 15.0 months, response rate of $19 \%){ }^{7}$ However, no third- or fourth-line treatment has been established after the failure of the abovementioned systemic agents. Recently, combination therapy of TKIs and ICPIs has been suggested as a promising strategy to improve treatment efficacy in advanced HCC patients based on the theory that TKIs may have immunemodulating effects. ICPIs can enhance either T cell activation
(anti-PD-1 and anti-PDL-1) or T cell priming (anti-cytotoxic T-lymphocyte-associated antigen 4). TKIs can complement ICPI therapy by enhancing tumor antigen expression, dendritic cell maturation and activity, immunogenic tumor cell death, and $\mathrm{T}$ cell infiltration into tumors. ${ }^{5}$ Currently, there are several ongoing trials that are conducting evaluations on the combination therapy of TKIs and ICPIs. For example, a trial on the combination of pembrolizumab and lenvatinib for HCC treatment was initiated and high response rates (50- 
70\%) have been reported. ${ }^{8}$

In conclusion, by combination therapy of nivolumab and sorafenib, we safely and effectively treated an advanced HCC patient showing disease progression after previous nivolumab monotherapy. To the best of our knowledge, no case of combined TKI and ICPI therapy overcoming ICPI resistance has been reported in Korea, and we herein offered our findings. We need to conduct further ongoing trials regarding the combination of TKIs and ICPIs.

\section{Conflicts of Interest}

The authors have no financial conflicts of interest.

\section{REFERENCES}

1. Cheng AL, Guan Z, Chen Z, Tsao CJ, Qin S, Kim JS, et al. Efficacy and safety of sorafenib in patients with advanced hepatocellular carcinoma according to baseline status: subset analyses of the phase III Sorafenib Asia-Pacific trial. Eur J Cancer 2012;48:14521465.

2. Bruix J, Raoul JL, Sherman M, Mazzaferro V, Bolondi L, Craxi A, et al. Efficacy and safety of sorafenib in patients with advanced hepatocellular carcinoma: subanalyses of a phase III trial. J Hepatol 2012; $57: 821-829$.

3. Bruix J, Qin S, Merle P, Granito A, Huang YH, Bodoky G, et al. Regorafenib for patients with hepatocellular carcinoma who progressed on sorafenib treatment (RESORCE): a randomised, doubleblind, placebo-controlled, phase 3 trial. Lancet 2017;389:56-66.

4. Marrero JA, Kulik LM, Sirlin CB, Zhu AX, Finn RS, Abecassis MM, et al. Diagnosis, staging, and management of hepatocellular carcinoma: 2018 practice guidance by the american association for the study of liver diseases. Hepatology 2018;68:723-750.

5. Hughes PE, Caenepeel S, Wu LC. Targeted therapy and checkpoint immunotherapy combinations for the treatment of cancer. Trends Immunol 2016;37:462-476.

6. Kudo M, Finn RS, Qin S, Han KH, Ikeda K, Piscaglia F, et al. Lenvatinib versus sorafenib in first-line treatment of patients with unresectable hepatocellular carcinoma: a randomised phase 3 noninferiority trial. Lancet 2018;391:1163-1173.

7. El-Khoueiry AB, Sangro B, Yau T, Crocenzi TS, Kudo M, Hsu C, et al. Nivolumab in patients with advanced hepatocellular carcinoma (CheckMate 040): an open-label, non-comparative, phase 1/2 dose escalation and expansion trial. Lancet 2017;389:2492-2502.

8. Kudo M. Combination cancer immunotherapy in hepatoceullar carcinoma. Liver Cancer 2018;7:20-27. 